\title{
Correlation between maternal eating disorder and early infant feeding regulation: a cross -sectional study
}

Rachel F. Rodgers ${ }^{1,2}$, Morgan Hines ${ }^{3}$, Alaina Martens ${ }^{3}$ and Emily Zimmerman ${ }^{3^{*}}$

\begin{abstract}
Background: The post-partum period is a vulnerable time for mothers in terms of eating disorder symptoms and is critical for the establishment of feeding patterns in infants. This study aimed to investigate the relationships between maternal eating disorder symptoms and objective indices of feeding regulation at 3 months, as well as perceived breastfeeding self-efficacy.

Methods: A sample of $n=73$ full-term mother-child dyads ( $44 \%$ female) participated in the study. Mothers selfreported eating disorder symptoms and breastfeeding self-efficacy and objective indices of infant feeding regulation were obtained in the home.

Results: Findings revealed the existence of relationships between higher maternal eating disorder symptoms, and objective indices of infant feeding regulation with substantial gender differences in the patterns emerging. Among mother-daughter dyads, maternal weight and shape concerns were associated with higher infant transfer volume and rate during bottle feeding. In contrast, among mother-son dyads, higher maternal eating disorder symptoms, including weight, shape, and eating concern, were associated with lower infant transfer volume and rate as well as lower levels of proficiency while taking their bottle.
\end{abstract}

Conclusion: Relationships emerged between higher maternal eating disorder symptoms and feeding regulation with substantial gender differences in these patterns. Additional research clarifying the underlying mechanisms of these associations is warranted and further efforts should be directed towards supporting mothers during the postpartum period.

Keywords: Postpartum, Feeding regulation, Mothers, Gender, Infant

\section{Background}

An estimated $25-45 \%$ of typically developing children experience some type of feeding problem [1-3]. Such difficulties are concerning as they may persist and influence child development as well as heighten the risk for future eating concerns in children and adolescents [4]. Previous

\footnotetext{
*Correspondence: e.zimmerman@neu.edu

${ }^{3}$ Speech and Neurodevelopment Lab, Department of Communication Sciences and Disorders, Northeastern University, 360 Huntington Avenue, Boston, MA 02115, USA

Full list of author information is available at the end of the article
}

studies have documented a relation between early infant feeding difficulties and greater maternal breastfeeding difficulties, and disordered eating behaviors $[1,5,6]$. However, the mechanisms underlying these relationships are not well understood.

Importantly, conceptualizations of feeding disorders have proposed that they be framed as relational disorders, thus acknowledging that infants feeding occurs within an interaction $[1,7]$. In this way, mothers with higher levels of eating concerns have also displayed lower levels of adaptation to their infants when feeding 
them [8], and may feel less comfortable breastfeeding. Thus, dyadic interactions during feeding may constitute an underlying mechanism for the relationship between maternal disordered eating and infant feeding difficulties. However, to date research in this area is scant. Moreover, the small amount of research that has been conducted among young infants during the first months postpartum has focused exclusively on food refusal as an outcome and has relied mainly on self-report or visual observational data for the feeding assessment $[6,8,9]$. The reliance on such methodologies limits the investigation of these mechanisms. One observational longitudinal study, however, suggested that sucking patterns in infancy predicted child pickiness between the ages of 3 and 5 years [4], highlighting the need for more work in this area.

The present study therefore sought to contribute to this area of research and investigate the relationships between maternal eating disorder symptoms and objective indices of feeding regulation at 3 months. In addition, maternal self-reported perceptions of feeding were assessed to contrast with objective indices. Given the previously documented gender-differences in feeding behaviors and practices as well as later outcomes [10, 11 , we examined relationships among mother-daughter and mother-son dyads separately. Given the exploratory nature of the study, no specific directional hypotheses were formulated.

\section{Methods}

\section{Aim, design, and setting}

The aim of the present study was to investigate the relationships between maternal eating disorder symptoms and objective indices of feeding regulation, as well as maternal perceived breastfeeding self-efficacy in fullterm infants at 3 months of age, examining these relationships between mother-daughter and mother-son dyads. We used a cross-sectional and correlational design with self-report and objective measures. The study was approved by the institutional review board (Protocol Number: 17-08-19). This study was completed in the greater Boston area with study visits occurring in participants' homes.

\section{Procedures}

Participants in this study were taken from a larger study that examined the relation between early sucking, oral feeding, and vocal development [12]. Parents consented for their own participation and that of their infants. Infants and their parents were recruited by word of mouth, Facebook groups, and flyer distribution. Caregivers were compensated with an Amazon gift card. Exclusion criteria for the broader study was infants born with congenital and/or chromosomal anomalies. The current study focused only on full-term infants at 3-months with primarily breastfeeding and only occasional bottle feeding (breastmilk/formula). Mothers and infants were included at 3 months post-partum as this is a period in which families typically have some established routine and feeding schedule and have not begun more complex oral movements. The study occurred in the infant's house approximately $1 \mathrm{~h}$ before the infant's scheduled feed as determined by the parent. During the study, the mother completed the eating disorder symptoms scale, the breastfeeding self-efficacy scale, and the researchers observed a bottle feed and completed the oral feeding skills (OFS) scale.

\section{Measures \\ Eating disorder symptoms}

The Eating Disorder Examination Questionnaire (EDEQ) 6.0 [13] focuses on the past 28 days and uses a 7-point rating scale ranging from 0 to 6 , to derive scores on four subscales that have been used in non-clinical populations (Restraint, Eating Concern, Weight Concern, and Shape Concern) [14]. Higher scores indicate higher symptom levels. Here, internal consistency was alpha $=.68$ for restraint, ${ }^{1} .83$ for weight concern, .91 for shape concern, and .73 for eating concern respectively.

\section{Breastfeeding self-efficacy}

Breastfeeding self-efficacy was assessed using the 14 item Breastfeeding Self-Efficacy Scale Short-Form [15]. Items begin with "I can always" and include statements such as "determine that my baby is getting enough milk." Participants rate their agreement using a 5-point scale ranging from 1 (not at all confident) to 5 (completely confident). Scores were summed with higher scores indicating greater breastfeeding self-efficacy. Here, internal consistency was .95.

\section{Feeding regulation}

Feeding regulation was determined using the OFS, developed by Lau and Smith [16] as a novel approach to assess oral feeding regulation of infants. While initially created to better define oral feeding metrics with preterm infants, the scale provides a simple means of measuring certain indices of oral feeding regulation among all bottle-fed infants. The OFS was completed while observing the caregiver offer their infant the bottle. If the infant was fed

\footnotetext{
${ }^{1}$ The alpha value for restraint was somewhat low, and inspection of the indices revealed that if item 2 were deleted, the alpha value would be $>.70$ and therefore acceptable. However, to preserve comparability of the findings here with other studies, the 5-item version of this scale was retained. Sensitivity analyses revealed that the findings were identical using the 4-item version of the Restraint scale with adequate internal reliability.
} 
Table 1 Descriptive statistics and gender differences for study variables

\begin{tabular}{|c|c|c|c|}
\hline & $\begin{array}{l}\text { Males } n=38 \\
\text { Median (range) }\end{array}$ & $\begin{array}{l}\text { Females } n=32 \\
\text { Median (range) }\end{array}$ & $P$-value \\
\hline \multicolumn{4}{|l|}{ Oral Feeding Skills } \\
\hline Initial Volume & 118.29 (29.57-273.56) & 118.29 (29.57-177.44) & .546 \\
\hline Transfer Volume & $73.50(.00-100.00)$ & $83.30(.00-100.00)$ & .505 \\
\hline Proficiency & $39.25(.00-85.73)$ & $35.41(.00-100.00)$ & .794 \\
\hline Transfer Rate & $8.80(.00-22.00)$ & $5.55(.00-20.33)$ & .375 \\
\hline \multicolumn{4}{|l|}{ Maternal Eating Concerns } \\
\hline Restraint & $.20(.00-3.20)$ & $.10(.00-3.40)$ & .472 \\
\hline Weight concern & $1.70(.00-4.00)$ & $1.20(.00-5.00)$ & .507 \\
\hline Shape concern & $1.62(.00-4.75)$ & $1.31(.00-4.87)$ & .825 \\
\hline Eating concern & $.20(.00-2.2)$ & $.20(.00-2.8)$ & .550 \\
\hline Global & $1.07(.00-3.28)$ & $.92(.00-3.46)$ & .908 \\
\hline Breastfeeding Self-Efficacy & $59.00(33.00-70.00)$ & $63.00(20.00-70.00)$ & 1.00 \\
\hline
\end{tabular}

Note: Mann Whitney was used for comparisons across sexes, ${ }^{*} p<.05$

breastmilk, the mother put her milk in the infant's bottle, otherwise they used their preferred formula. No instructions were given regarding the amount of milk to provide. During the observation, a researcher denoted the following outcomes: initial volume $(\mathrm{ml})$, transfer volume, (\% volume taken/total volume to be taken), proficiency (\% volume taken during the first $5 \mathrm{~min} /$ total volume prescribed), and transfer rate $(\mathrm{ml} / \mathrm{min})$.

\section{Statistical analyses}

Given that the distribution of the EDE-Q subscales was somewhat skewed in this small, non-clinical sample, Spearman correlations were conducted. For sensitivity purposes, partial Pearson correlations controlling for infant weight were also conducted. Given the small sample size, and the exploratory nature of these data, while statistical significance $(p<.05)$ is reported, effect sizes were interpreted such that coefficients between .10 and .29 represent a small association, coefficients between .30 and .49 represent a medium association, and coefficients of .50 and above represent a large association or relationship [17].

\section{Results}

Data from a sample of $n=73$ full-term primarily breastfeeding mother-child dyads ( $44 \%$ female), with an average birth weight of $3.44 \mathrm{~kg}(\mathrm{SD}=.44)$, who were participating in a larger study were included. Mothers were on average 33.68 years old $(S D=3.59)$, with infants aged 3.02 months $(\mathrm{SD}=.30)$, and half of the sample $(49 \%)$ were firstborns. In our cohort, $97 \%$ of parents were married, $77 \%$ were employed fulltime, and $90 \%$ had a college or graduate degree.
Mann Whitney tests revealed no significant differences between males and females across the dependent measures (see Table 1). In addition, no differences were found on variables of interest between firstborns and infants with siblings (data not shown). Among motherdaughter dyads, spearman correlations revealed that selfreported maternal weight concerns and shape concerns were moderately associated with higher transfer volume and transfer rate (see Table 2). Among mother-son dyads a different pattern emerged, with maternal weight and shape concerns moderately associated with lower transfer volume, transfer rate, and proficiency. In addition, among mother-son dyads, greater maternal eating concerns were moderately associated with lower transfer rate and proficiency. Breastfeeding self-efficacy revealed no relationships with feeding regulation while taking a bottle, with the exception of a small relationship with lower transfer rate among mother-daughter dyads. Sensitivity analyses revealed no substantial differences in the patterns of association.

\section{Discussion}

The aim of the present study was to investigate the relationships between maternal eating disorder symptoms, objective indices of feeding regulation, and maternal perceived breastfeeding self-efficacy among 3-monthold mother-daughter and mother-son dyads. Overall, findings suggest the existence of relationships between higher maternal eating disorder symptoms and feeding regulation. Moreover, substantial gender differences in these patterns emerged. Among mother-daughter dyads, as indicated by the positive correlation, maternal weight and shape concerns were associated with higher levels of infant transfer volume and rate, indicating that mothers 
Table 2 Bivariate associations among study variables

\begin{tabular}{|c|c|c|c|c|c|c|c|c|}
\hline & \multicolumn{8}{|c|}{ Oral Feeding Skills } \\
\hline & \multicolumn{2}{|c|}{ Total Volume } & \multicolumn{2}{|c|}{ Transfer Volume } & \multicolumn{2}{|c|}{ Proficiency } & \multicolumn{2}{|c|}{ Transfer Rate } \\
\hline & Girls & Boys & Girls & Boys & Girls & Boys & Girls & Boys \\
\hline \multicolumn{9}{|l|}{ Maternal Eating Concerns } \\
\hline Restraint & .05 & .26 & .13 & .10 & .01 & .31 & .17 & .27 \\
\hline Weight concern & -.03 & -.06 & $.34^{\natural}$ & $-.38^{\natural}$ & .21 & $-.39 *$ & $.33^{\natural}$ & $-.43^{*}$ \\
\hline Shape concern & -.05 & -.04 & $.43^{*}$ & -.29 & .30 & $-.35^{\natural}$ & $.43^{*}$ & $-.37^{n}$ \\
\hline Eating concerns & .05 & -.04 & .22 & -.14 & .06 & $-.41^{*}$ & .25 & $-.39^{4}$ \\
\hline Breastfeeding self-efficacy & -.14 & .12 & -.16 & .03 & -.13 & .17 & -.25 & .12 \\
\hline
\end{tabular}

${ }^{*} p<.05,{ }^{n} p<.10$

with higher weight and shape concerns had daughters who ate more and faster, than those with lower weight and shape concerns. In contrast, among mother-son dyads, as indicated by the negative correlation, higher maternal eating disorder symptoms, including weight, shape, and eating concerns, were associated with lower levels of infant transfer volume and rate as well as lower levels of proficiency. These findings suggest that in these dyads higher maternal concerns co-occurred with sons feeding slower, less proficiently and overall eating less.

To our knowledge this is the first time the maternal eating concerns and infant feeding regulation have been examined across mother-son and mother-daughter dyads at this early time point. These findings extend the aforementioned relations between maternal disordered eating behaviors and specific infant feeding patterns including greater feeding difficulties found in previous studies [1, 6] by documenting feeding regulation in young infants. Furthermore, this study is unique in its contribution by revealing gender differences in the patterns of these relations. These gender differences are difficult to interpret in correlational data, and in the absence of other indicators such as feeding frequency. Given that no gender differences emerged in the median levels of the observed variables, it seems unlikely that infants were reacting to differences in the amount of milk provided by mothers to sons compared to daughters. It is possible that infant hunger due to differences in feeding frequency across genders might contribute to these patterns. Indeed, it has been suggested that parental concern about child weight and feeding patterns may reveal gender-biases, such that parents are more concerned about their young daughters' weight [18] and that eating pathology has been associated with more controlling feeding practices in mothers of 3-year old daughters, but not sons [19]. However, it is notable that the pattern of associations among motherson dyads was more consistent across indices of maternal disordered eating and feeding regulation compared to the pattern among mother-daughter dyads. Given this, it might be possible that among mothers with sons, associations between infant feeding regulation and maternal disordered eating is evident among dyads with higher levels of difficulty, characterized by maternal concerns including difficulty regulating eating behaviors, and feeding difficulties respectively. In contrast, it may be that among mother-daughter dyads, patterns are evident across lower levels of concerns and mainly reflect associations with body image concerns that could be more normative in the postpartum period. Our data suggest that this is likely a bidirectional relationship underlying these patterns that begin early in infancy and warrant future investigation across the lifespan and across feeding development.

Although the pathway is not yet clear, these relationships could be nested in the complexities of the motherdaughter relationship, as well as gendered expectations regarding eating, weight, and shape. Indeed, research has shown that mothers tend to report being more concerned about their daughters' weight compared to that of their sons [20]. These differences likely reflect broader social differences in weight bias and overvaluation of weight and shape, with young women experiencing greater appearance pressures as compared to their male counterparts [21]. However, perhaps relatedly, research has shown that mothers perceive hunger cues differently among their breastfed female infants as compared to their male infants [22]. Therefore, the differences observed may also partly be the result of maternal feeding practices in response to their perceptions of children's hunger cues, as well as their socialized beliefs around gender differences in those.

Interestingly, with the exception of transfer rate among female infants that revealed a small effect associated with lower maternal breastfeeding self-efficacy, no other patterns between feeding regulation and maternal perceived breastfeeding self-efficacy emerged. This is important as it suggests that while breastfeeding self-efficacy is 
associated with some infant feeding measures, such as infant latch and infant cues, it may not be associated with actual feeding proficiencies. Such a finding is consistent with the disconnect observed between maternal perceptions and feeding practices among older children [23].

This study presents a number of limitations including the cross-sectional and correlational design, the lack of maternal anthropomorphic data, and the somewhat small sample size. In addition, it was not possible to control for the time since last feeding, although parents scheduled the appointment for approximately $1 \mathrm{~h}$ before the predicted feeding time. Nevertheless, our study makes an important contribution to the existing literature by representing the first investigation of the relationships between maternal eating disorder symptoms and feeding regulation in very young infants.

\section{Conclusion}

Our findings suggest that such relationships exist, although reveal interesting gender differences. Additional research confirming these patterns and investigating the underlying mechanisms is warranted. While the interpretation of these findings requires further investigation, they highlight the need for psychosocial support for mothers during the postpartum period, which is a vulnerable time for mothers who may experience heightened body image and eating concerns [24]. Furthermore, as our data suggest that maternal breastfeeding self-efficacy may be independent of their infants' feeding behaviors, strategies to enhance breastfeeding confidence should be targeted toward the mother's perception of the breastfeeding experience and incorporated in intervention efforts and resources.

\section{Abbreviations}

OFS scale: The Oral Feeding Skills; EDE-Q: The Eating Disorder Examination Questionnaire.

\section{Acknowledgements}

We would like to thank the caregivers and infants who participated.

\section{Authors' contributions}

RR was responsible for the interpretation of data and the drafting and revision of the manuscript. MH was responsible for the acquisition of the data, the statistical analysis, and the revision of the manuscript. AM was responsible for the acquisition of the data, the interpretation of data, and the revision of the manuscript. EZ was responsible for the conception and design of the study, the analysis and interpretation of the data, as well as the initial draft and substantial revision of the manuscript. All authors agreed to the manuscript in its submitted form. All authors agree to be accountable for their own contributions and agree to investigate any questions about the manuscript's accuracy or integrity thoroughly with evidence of resolution stated in the manuscript.

\section{Funding}

This study was funded by the NIH R21DC016030. The funding body had no role in the design, data collection, analysis, interpretation of data, or the writing of this manuscript.

\section{Availability of data and materials}

The data that support the findings of this study are available from the corresponding author upon reasonable request.

\section{Declarations}

\section{Ethics approval and consent to participate}

The study was approved by the institutional review board at Northeastern University (Protocol Number: 17-08-19). All methods were performed in accordance with the relevant institutional review board guidelines and regulations. Informed consent was obtained from all participants or, if participants are under 16 , from a parent and/or legal guardian.

\section{Consent for publication}

Not applicable.

\section{Competing interests}

The authors declare that they have no competing interests.

\section{Author details}

'APPEAR, Department of Applied Educational Psychology, Northeastern University, 360 Huntington Avenue, Boston, MA 02115, USA. ${ }^{2}$ Department of Psychiatric Emergency \& Acute Care, Lapeyronie Hospital, CHRU Montpellier, 291 Av. du Doyen Gaston Giraud, 34000 Montpellier, France. ${ }^{3}$ Speech and Neurodevelopment Lab, Department of Communication Sciences and Disorders, Northeastern University, 360 Huntington Avenue, Boston, MA 02115, USA.

Received: 15 January 2021 Accepted: 6 December 2021

Published online: 20 December 2021

\section{References}

1. Bryant-Waugh R, Markham L, Kreipe RE, Walsh BT. Feeding and eating disorders in childhood. Int J Eat Disord. 2010;43(2):98-111.

2. Estrem HH, Pados BF, Park J, Knafl KA, Thoyre SM. Feeding problems in infancy and early childhood: evolutionary concept analysis. J Adv Nurs. 2017;73(1):56-70.

3. Zimmerman E, Rosner A. Feeding swallowing difficulties in the first three years of life: a preterm and full-term infant comparison. J Neonatal Nurs. 2018;24(6):331-5

4. Jacobi C, Agras WS, Bryson S, Hammer LD. Behavioral validation, precursors, and concomitants of picky eating in childhood. J Am Acad Child Adolesc Psychiatry. 2003:42(1):76-84.

5. Martini MG, Barona-Martinez M, Micali N. Eating disorders mothers and their children: a systematic review of the literature. Arch Womens Ment Health. 2020;23(4):449-67.

6. Micali N, Simonoff E, Stahl D, Treasure J. Maternal eating disorders and infant feeding difficulties: maternal and child mediators in a longitudinal general population study. J Child Psychol Psychiatry. 2011;52(7):800-7.

7. Freud A. The psychoanalytic study of infantile feeding disturbances. Psychoanal Study Child. 1946;2:119-32.

8. Squires C, Lalanne C, Murday N, Simoglou V, Vaivre-Douret L. The influence of eating disorders on mothers' sensitivity and adaptation during feeding: a longitudinal observational study. BMC Pregnancy Childbirth. 2014;14(1):274.

9. Farrow C, Blissett J. Maternal cognitions, psychopathologic symptoms, and infant temperament as predictors of early infant feeding problems: a longitudinal study. Int J Eat Disord. 2006;39(2):128-34.

10. Cable N, Bartley M, McMunn A, Kelly Y. Gender differences in the effect of breastfeeding on adult psychological well-being. Eur J Pub Health. 2012;22(5):653-8.

11. Farrow C, Blissett J. Breast-feeding, maternal feeding practices and mealtime negativity at one year. Appetite. 2006;46(1):49-56.

12. Martens A, Hines M, Zimmerman E. Changes in non-nutritive suck between 3 and 12 months. Early Hum Dev. 2020;149:105141.

13. Fairburn CG, Cooper Z, O'Connor M. Eating disorder examination (16.0D). In: Fairburn CG, editor. Cognitive behavior therapy and eating disorders. New York: Guilford Press; 2008. 
14. Mond JM, Myers TC, Crosby RD, Hay PJ, Rodgers B, Morgan JF, et al. Screening for eating disorders in primary care: EDE-Q versus SCOFF. Behav Res Ther. 2008;46(5):612-22.

15. Dennis $C L$. The breastfeeding self-efficacy scale: psychometric assessment of the short form. J Obstet Gynecol Neonatal Nurs. 2003;32(6):734-44.

16. Lau C, Smith EO. A novel approach to assess oral feeding skills of preterm infants. Neonatology. 2011;100(1):64-70.

17. Cohen J. A power primer. Psychol Bull. 1992;112(1):155-9.

18. Peyer KL, Welk G, Bailey-Davis L, Yang S, Kim J-K. Factors associated with parent concern for child weight and parenting behaviors. Child Obes. 2015;11(3):269-74.

19. Blissett J, Meyer C, Haycraft E. Maternal and paternal controlling feeding practices with male and female children. Appetite. 2006;47(2):212-9.

20. Campbell MWC, Williams J, Hampton A, Wake M. Maternal concern and perceptions of overweight in Australian preschool-aged children. Med $J$ Aust. 2006;184(6):274-7.

21. Fikkan $J L$, Rothblum ED. Is fat a feminist issue? Exploring the gendered nature of weight bias. Sex Roles. 2012;66(9):575-92.

22. Wright P. Mothers' assessment of hunger in relation to meal size in breastfed infants. J Reprod Infant Psychol. 1987;5(3):173-81.

23. Bergmeier $\mathrm{HJ}$, Skouteris $\mathrm{H}$, Hetherington MM, Rodgers RF, Campbell $\mathrm{KJ}$, Cox R. Do maternal perceptions of child eating and feeding help to explain the disconnect between reported and observed feeding practices?: a follow-up study. Matern Child Nutr. 2017;13(4):n/a.

24. Rodgers RF, O'Flynn JL, Bourdeau A, Zimmerman E. A biopsychosocial model of body image, disordered eating, and breastfeeding among postpartum women. Appetite. 2018;126:163-8.

\section{Publisher's Note}

Springer Nature remains neutral with regard to jurisdictional claims in published maps and institutional affiliations.

- fast, convenient online submission

- thorough peer review by experienced researchers in your field

- rapid publication on acceptance

- support for research data, including large and complex data types

- gold Open Access which fosters wider collaboration and increased citations

- maximum visibility for your research: over $100 \mathrm{M}$ website views per year

At BMC, research is always in progress.

Learn more biomedcentral.com/submissions 